\title{
Household food insecurity, copying strategies and its associated with parents food preference to children in Dabat Demographic and health surveillance system; North West Ethiopia, April 2019
}

nigusie birhan tebeje ( $\nabla$ nigusiebirhan@gmail.com )

University of Gondar, Ethiopia

Gashaw Andargie Biks

University of Gondar College of Medicine and Health Sciences

Solomon Mekonnen Abebe

University of Gondar College of Medicine and Health Sciences

Melike Endris Yesuf

University of Gondar College of Medicine and Health Sciences

Research article

Keywords: food insecurity, household, Dabat, surveillance, Gondar and Ethiopia

Posted Date: July 28th, 2019

DOI: https://doi.org/10.21203/rs.2.12009/v1

License: (9) This work is licensed under a Creative Commons Attribution 4.0 International License. Read Full License 


\section{Abstract}

Abstract Introduction:- the association of household food insecurity with a physical health problem, quality of life, mental health problem and utilization of health service was reported by several studies. However, the association of household food insecurity with parent's food preference to children is not assessed even though all of these factors may make parents to prefer inappropriate child food. Therefore this study was aimed to determine the association of food insecurity with parent's food preference. Methods and materials: - A community-based cross-sectional survey was conducted among 6215 households in Dabat district. Data were collected and supervised by experienced data collectors and supervisors working for the surveillance site after five days of training on the tool. Data were entered into the epi data template then transported to Stata 14 software for analysis. An ordinal logistic regression model was fitted to identify predictor for household food insecurity. Finding: About $21.77 \%$ of the households were food insecured in Dabat district. High wealth 61\% (57-80\%), and achieving college education $97 \%(74-99.6 \%)$ were associated with the less likelihood of household food insecurity. While mothers/caretakers preference to feed children always fruit and vegetable only 1.75(1.11-2.57) and, always milk and meat only 1.72(1.17-2.52) were associated were the more likelihood of experiencing the worst state of household food insecurity. Conclusion: -A significant number of households in Dabat had experienced household food insecurity associated with wealth status of the household, and, parents/caretakers specific type of food preference for their children. Therefore we recommend counseling services on the importance of feeding a child with a balanced diet in the improvement of household food security. Keywords: - food insecurity, household, Dabat, surveillance, Gondar and Ethiopia

\section{Background}

Food security is a broad concept that entails means to produce own agricultural subsistence or ability to purchase food for a population to be healthy and well-nourished. It exists when all people at all times have physical, social and economic access to sufficient, safe and nutritious food that meets their dietary needs and food preferences for an active life. (1). Experiencing insufficiency of food, dietary diversity or being vulnerable to this risk is food insecurity (2).

Food insecurity is a devastating situation manifested with under-nutrition, hidden hunger, obesity, and overweight. In Africa, the problem of food insecurity was widely distributed and remained unsolved since the 1970s (3). Even though there are encouraging efforts against food insecurity African households had experienced the very high magnitude of household food insecurity at present. In poor provinces of South Africa, nearly $68 \%$ of households had experienced a severe form of food insecurity (4).

Ethiopia remains one of the ten poorest countries in the world $(5,6)$. About $83 \%$ of Ethiopia population is rural resident whose economy s depends on agriculture most vulnerable for the adverse effects of climatic change. (7). After a widespread of Eli NINO 10.2 million people food insecure in Ethiopia because of livestock death and drops in crop production capacities $(8,9)$. 
as to the studies, the magnitude of household food insecurity in Ethiopia ranges from $38.7 \%$ to $82.3 \%$ with a trend shifting from urban to rural households (10-15). In addition to this poverty was widely distributed particularly in a rural community which is considered as the immediate cause of food insecurity. (16).

Household food insecurity was reported to be associated with mental health problems like anxiety, depression, neurologic disorder (13, 17-22), chronic illness affecting maternal health (23-26) maternal malnutrition, increased maternal body mass index and pregnancy complications (27-30), reduced dietary diversity $(21,31,32)$ and transgenerational exposure to violence a risk for stress, family trauma, depression and problems with emotional management (33) lower quality of life (34), low contraceptive utilization rate (35), early age at marriage. All of these factors have had a significant effect on parental food preference for under-five children but the association is not assessed yet.

In Ethiopia about $40 \%$ of children under the age of five were stunted, $9 \%$ were wasted, $25 \%$ were underweighted and about $3 \%$ were overweighted or obese in Ethiopia in 2014(36). Overall 56\% of children were suffering from anemia, $46 \%$ of preschool children were vitamin A deficient $(37,38)$ probably due to household food insecurity and parents food preference. However, the association between household food insecurity and parental food preference is not investigated. Therefore the aim of this study was estimating the magnitude of household food insecurity and its association with parental food preference for their under-five children.

\section{Methods}

Study area: - The study was conducted in Dabat Demographic and Health Surveillance system site (DHSS). The Dabat DHSS covers 13 kebeles (four urban and 9 rural) divided into 83 clusters. The altitude of the district is divided into highland, Midland, and lowland climatic conditions. Dabat town, the capital of the district, is located at about $821 \mathrm{Km}$ from Addis Ababa and $75 \mathrm{Km}$ from Gondar town to the north. The district has six health centers, three health stations, and thirty-one health posts providing health services to the community. The total population of the district in 2012/13 was estimated to be 158,250 of whom about 70,611 people were the population of HDSS site with a sex ratio nearly 1:1. in the HDSS there are 7,918 under-five children from 6,314 households included in this study (39).

Study design and population: the community-based cross-sectional study design was carried out among rural and urban households of HDSS site from April to December 2016. Heads households in the HDSS site during the study period were study participants. In the absence of heads of the household during visit day; mother or other senior household members beyond 18 years were interviewed for the tool after consenting.

Data collection tool and data collection procedure: -a pre-tested interviewer-administered structured questionnaire prepared with local language was used to collect data on socio-demographic, maternal and child health service utilization and mothers/caretakers food preference habits for their under-five children. FANTA III food insecurity access measurement scale version 3 was used to collect data. A five-day 
intensive training was provided for 15 data collectors and 5 supervisors. A pre-test was conducted in the rural and urban kebeles of Dabat district which are not included in the HDSS and necessary modification of the tool was made according to the inputs obtained from the pre-test.

Data processing and analysis: collected data were entered into Epi data template prepared by Amharic language having similar content with the data collection question to avoid data entry error related to the translation of the responses. Data entry was performed by five experienced data entry clerks working for Dabat HDSS. The overall data entry process was supervised by the data manager working HDSS. Entered data was transported to STATA version 14.1 software. Before actual data analysis data management and clearance was performed by the data manager. Before fitting univariate and multivariate logistic regression models the relationship of the dependent variable with each independent variables was assessed by Pearson's chi-square test. Then variables with significant Pearson's chi-square were considered for the univariate ordinal logistic regression model. All variables significant at the univariate ordinal logistic regression model were fitted into multivariate ordinal logistic regression models to identify predictors for the worsening state of household food insecurity. Proportional or cumulative odds ratio assumption was checked by significant Pearson's chi-square.

Study variables

Dependent variable: - state of household food insecurity

\section{Independent variables}

Socio-demographic characters: - (wealth status of the household, educational status, marital status and residence of head of the household number of children in the household, age of children in the household, sex of children in the household, family size, religion ethnicity, occupation and availability of garden and source of food item for the household )

Maternal and child health utilization: - (TT vaccine, Iron supplementation, ANC visit, and Place delivery for the last pregnancy, PNC service for the last birth, VitA supplementation for children in household, Child deworming, BCG, polio, DPT, Penta Valiant, Rota ,PCV , measles vaccine, illness and Treatment for illness )

Food preference related factors: - (preference of balanced diet, specific food preference, and preference of family food)

\section{Operational definition}

Household food security:-if the interviewed heads of the household answered rarely for only Q1a

Mild food insecurity:- if the interviewed heads of the household answered sometimes or often for Q1a or rarely, sometimes or often for Q2a or rarely for Q3a or Q4a it was considered as mild household food insecurity. 
Moderate food insecurity: - if the interviewed heads of the household answered sometimes or often for Q3a or Q4a or rarely, sometimes Q5a or Q6a it was considered as moderate household food insecurity.

Sever food insecurity:- if the interviewed heads of the household answered often for Q5a or Q6a or rarely, sometimes or often for questions Q7a-Q9a it was considered as severe household food insecurity

Food preference:- if parents choose to feed food with the same caloric content more than once per day it is considered as preferring to feed specific food preference, if they tend to feed any available food or the food prepared for adult family members it is considered as preference to feed family food and if there is habit of balancing child food from locally available food items it is preference to feed balanced diet.

\section{Results}

\section{Socio-demographic characteristics of study participants}

Of the total 6215 heads of the household in the study area, 5724 had completely responded for the survey making the response rate $92.1 \%$. The majority (81.96\%) of the study participants were male and almost all $(98.74 \%)$ of them were Amhara in their ethnicity. More than half $(55.76 \%)$ of the participants were unable to read and write. More than three fourth $(76.53 \%)$ of them were married. Nearly half (47.20\%) participants were farmers by occupation and $34.02 \%$ were from households with low wealth status. The majority $(80.50 \%)$ of participants were rural residents where $32.38 \%$ of households have six and more family members (Table 1 ).

\section{Maternal and child health service utilization among households in Dabat HDSS site}

Data on maternal and child health service utilization were collected from 5724 women 6521 under-five children during the study period. Two-third (65.86\%) of women have a history of ANC visit during pregnancy. About $69.81 \%$ of deliveries were home deliveries and $8.79 \%$ of women have reported receiving of postnatal care. About $88.49 \%, 8.02 \%$ and $76.95 \%$ of women have received iron, vitamin $A$ supplementation and TT vaccine respectively. Regarding child health service utilization $54.01 \%, 37.05 \%$ and $95.67 \%$ under-five children have received vitamin A supplementation, deworming and at least one vaccine respectively. The survey result generally demonstrated decreasing vaccine coverage across the schedules (table 2).

\section{Under-five children health problems among households in Dabat HDSS site}

About 2702 under-five children have experienced health problem with in the last two weeks of the survey. Major health problem among under-five children reported by the parents/caretakers were febrile illness, watery diarrhea, and respiratory tract infection respectively (Fig 1).

\section{Parental food preference for under-five children}


Among the survey households 3577 (62.53\%), 941(16.44\%) and 1203(21.03\%) parents prefer to feed their under-five children with a family food, a specific type of food and balanced diet for their children, respectively. Among parents who prefer to feed a child with a specific type of food almost all $(87.53 \%)$ prefers to feed cereals and grains because their children like to eat

\section{Household food insecurity and its coping strategies in Dabat HDSS site}

About $21.77 \%$ of households were food in secured in Dabat HDSS within one month of the survey. More than half (50.24\%) food in-secured households reduce the amount of food as a coping strategy (fig 2 and fig 3).

\section{Factors associated with the increased magnitude of household food insecurity}

At the univariate ordinal logistic regression model heads of the household currently divorced 2.41(1.57-371), and no BCG vaccine 3.00(2.60-3.50), no vitamin A supplementation 2.67(2.55-3.03), no Rota III 2.15(1.76-2.63) and preferring to feed only fruit and vegetable 2.00(1.40-2.86) for children were variables strong associated with experiencing different levels of food insecurity at the household level. After adjustment of possible confounders at multivariate ordinal logistic regression model' variables like no BCG vaccine 1.85 (1.13-3.01) no vitamin A supplementation 2.03(1.34-3.10) and preferring to feed only fruit and vegetable for children 1.72(1.17-2.52) were found to be associated with the more likelihood of experiencing different levels of household food insecurity (table3).

\section{Discussion}

This study has determined the magnitude o of household food insecurity, major coping strategies, and associated factors in Dabat district North West Ethiopia. In this survey prevalence of household food insecurity was $21.77 \%$; of which $31 \%, 60 \%$, and $9 \%$ of the households had experienced mild, moderate and severe household food insecurity in Dabat district. The magnitude of household food insecurity in this survey was lower than the finding of studies in south Ethiopia where $82.3 \%$ of households were reported to be food insecurity (10), northeast and northwest Ethiopia which reported $55.3 \%$ and $70.7 \%$ of household food insecurity $(11,15)$. Similarly, household food insecurity southeast Ethiopia was $38.7 \%$ of (34), in Addis Ababa was 75\% (40)and southwest Ethiopia was $75.8 \%$ which was higher than the finding of our survey(41). This variation in magnitude of household food insecurity between the current survey and the above studies might be attributed to differences in the study setting, study period and the seasonal variation in food insecurity problems.

In our survey skipping or reducing meal size, borrowing money, selling a household asset, food or money aid and migratory work were major food insecurity coping strategies. The finding of our survey was in line with the finding of the studies carried out in Addis Ababa and Jimma Ethiopia $(41,42)$.

Households with medium wealth (42\% (24-45\%)) and high wealth status ( $71 \%(57-80 \%))$ in our study were less likely to experience the worst state of household food insecurity compared with households 
with low wealth status which was supported by the findings of different studies $(11,43,44)$. This inverse association between better household wealth status and experiencing any level of household food insecurity would be explained by the fact that household with better wealth would easily able to maintain food availability, sustainability, accessibility and utilization at the household level; the dimension of food security.

Households headed by those who achievement of primary education (33\% (10-55\%), secondary education (63\% (38-78\%) and college education (97\%(74-99.6\%) were less likely to experience any level of household food insecurity compared with households headed by those who were unable to read and write and this finding was supported the findings of other studies $(15,41,45,46)$. This inverse association between household food insecurity and better educational achievement of heads of the household might be attributed to improving the accessing of the information that helped to improve household productivity and better economic earnings that has a direct effect on household food insecurity.

Occupation of the head of the household was another factor significantly associated with household food insecurity. In this study, the head of household who was a farmer and private employee were $19 \%$ and $63 \%$ respectively less likely to experience household food insecurity compared with heads of household who are under age. This finding was supported by similar studies in North West and urban Ethiopia $(12,15,41)$. The highest risk of experiencing food insecurity by households headed by those who are youth can be explained by the fact that youth heads of the household were more likely to be less empowered in every aspect of their life which harm household productivity and household food insecurity.

Households with children who do not receive BCG vaccine 1.85(1.13-3.01) and vitamin A supplementation 2.03(1.34-310) more likely to experience any level of household food insecurity than households with children who receive BCG vaccine and vitamin A supplementation. This finding was in line with the findings of the studies in southern Ethiopia $(47,48)$. The positive association between experiencing any level of household food insecurity and do not receiving of BCG vaccine and vitamin A supplementation may be explained by the fact that those households with children who do not receive BCG and vitamin A may likely experience childhood illness which intern has direct relationship on household food insecurity by reducing food expenditure and increase health care costs which have direct effect on household food insecurity $(40,49)$.

Households with parents having a habit of preferring to feed fruit and vegetable only $1.70(1.11-2.57)$ and, milk and meat only for their children 1.72(1.17-2.52) were more likely to experience any level of household food insecurity than households with a parent who prefers to balance diet for their children. This positive association between preferring to feed specific type of food for children and household food insecurity may probably due to the worsen state of child health-related to problems in achieving minimum food diversity which has effect on child health that increase household health care expenses, and reduces household income which negatively affects household food security (31). 


\section{Conclusion}

A significant number of households in Dabat had experienced household food insecurity associated with wealth status of the household, educational status and occupation of the head of the household, maternal and child health service utilization and parents' specific food preference for their under-five children. Therefore we recommend empowerment and access of education, accessing of maternal and child health services in the community and arrangement counseling on the importance of balanced diet for under-five children in the community in addition to governmental efforts to improve agricultural productivity.

\section{List Of Abbreviations}

MDG Millennium Development Goal

IQ Intelligent Quotient

HIV/AIDS Human immune virus/Acquired immunodeficiency syndrome

HDSS Health and Demographic Surveillance System

KM Kilometer

PNC Postnatal care

ANC Antenatal care

\section{Declarations}

\section{Ethical approval and consent to participate}

Ethical clearance was obtained from the Institutional Ethical Review Board (IRB) of the University of Gondar with the reference no of R.NO.O/V/P/RCS/05/1220/2016. Informed verbal consent was obtained from the participants before the interview. The interviewers explained the Objective, benefit, and risk of the study before getting consent from parents of study participants. Besides, data collectors were instructed to assure the rights of the respondents to refuse or withdraw from the interview at any time without any form of prejudice. Children with undernutrition (mid-upper arm circumference (MUAC) $=11.5 \mathrm{~cm}$ or yellow), anemia and intestinal parasitosis were referred to nearest health facilities and health/nutritional education was also given to parents/caretakers by data collectors and supervisors. Confidentiality of the information was maintained by coding of all personal or household identifiers

\section{Consent for Publication}

The manuscript does not contain individual data with any personal identification. But the proposal with evaluated by the institutional ethical board to grant consent for publication. 
Availability of Data and materials

The datasets used and/or analyzed during the current study are available from the corresponding author on reasonable request.

\section{Competing interests}

We declare that we do not have a competing interest

\section{Funding}

The University of Gondar has funded the whole data collection and supervision activities of this project.

\section{Author's contribution}

NBT Participated in the conception, designs the study, analyzed the data and drafted the manuscript. GAB reviewed and approved the proposal and the manuscript, SMA reviewed and approved the proposal and the manuscript ME; reviewed and approved the proposal and the manuscript. All authors read and approve the final manuscript

\section{Acknowledgment}

We acknowledge mothers/caretakers of under-five children and data collectors. The project was funded by the University of Gondar

\section{Author's information}

NBT: Ph.D. Student: - University of Gondar; college of medicine and health sciences institute of public health.

GAB: associate professor of public and child health; Director Institute of public health, University of Gondar.

SMA: associate professor of nutrition, coordinator of master card foundation, university of Gondar MEY: professor of nutrition university of Gondar

\section{Limitation of the study}

Data were collected only from mothers/caretakers where involvement of both parents may better supplement the evidence.

\section{Strength of the study}

For assessment of mothers/caretakers food preference, the study considers a relatively large sample size and advanced statistical model. 


\section{References}

1. Coleman-Jensen A, Christian Gregory, and et. al. Household Food Security in the United States in 2013, ERR-173, US Department of Agriculture, Economic Research Service, September 2014.(173).

2. Caswell M, V.E. Méndez \& C.M. Bacon Food security and smallholder coffee production: current issues and future directions. ARLG Policy Brief \# 1. Agroecology and Rural Livelihoods Group (ARLG), University of Vermont Burlington, VT, USA 2012.

3. McLachlan M. DELIVERING ON THE COMMITMENT TO ERADICATE MALNUTRITION IN ALL ITS FORMS: THE ROLE OF THE UN SYSTEM. In: nutrition Uiawo, editor.: United nations 2015.

4. Zhou LMVMAMFAL. Household food insecurity in the poorest province of South Africa: level, causes, and coping strategies. Food Sec 2015 7:647-55.

5. BUREAU PR. WORLD POPULATION DATA SHEET WITH A SPECIAL FOCUS ON HUMAN NEEDS AND SUSTAINABLE RESOURCES. 2016 WORLD POPULATION DATA SHEET. 2016.

6. Stiftung B. BTI 2016 - Ethiopia Country Report. Gütersloh: Bertelsmann Stiftung. 2016.

7. Canada O. Country Profile: Ethiopia. December 2012.

8. Shitarek T. Ethiopia Country Report. 2012.

9. Allahoury CHALLENGES FACING AGRICULTURE AND FOOD SECURITY SITUATION REPORT food and agricultural organization of united nations 2016.

10. Stoecker NRaBJ. Household food insecurity and hunger among households in Sidama district, southern Ethiopia. Public Health Nutrition. 2011;15(7 ):1276-83.

11. Achenef Motbainor AWaAK. Level and determinants of food insecurity in East and West Gojjam zones of Amhara Region, Ethiopia: a community based comparative cross-sectional study. BMC Public Health 2016;16(503 ).

12. Craig Hadley DAL, Tefera Belachew, and et. al. Household capacities, vulnerabilities, and food insecurity: Shifts in food insecurity in urban and rural Ethiopia during the 2008 food crisis. Soc Sci Med 2011; 73(10):1534-42

13. Markos Tesfaye PK, Mette Frahm Olsen, and et. al. Food insecurity, mental health and quality of life among people living with HIV commencing antiretroviral treatment in Ethiopia: a cross-sectional study. Health and Quality of Life Outcomes 2016 14(37).

14. Tefera Belachew DL, Abebe Gebremariam, and et. al. Predictors of chronic food insecurity among adolescents in Southwest Ethiopia: a longitudinal study. BMC Public Health2012, 2012;12(604).

15. Worku Endale ZBM, Azeb Atinafu, and et. al. Food Insecurity in Farta District, Northwest Ethiopia: a community-based cross-sectional study. BMC Research Notes. 2014;7(130).

16. Ethiopia CSA. Ethiopia Demographic and Health Survey 2011. ICF International. 2012

17. C Hadley AT, F Tessema, and et. al. Food insecurity, stressful life events and symptoms of anxiety and depression in east Africa: evidence from the Gilgel Gibe growth and development study. J Epidemiol Community Health. 2008;62:980-6. 
18. E-S. Household food insecurity and symptoms of the neurologic disorder in Ethiopia: An observational analysis. BMC Public Health. 2010;10(802).

19. Mulusew G. Jebena MT, and et. al. Household food insecurity and mental distress among pregnant women in Southwestern Ethiopia: a cross-sectional study design. BMC Pregnancy and Childbirth 2015;15(250).

20. Shifferaw KMaS. CYCLES OF POVERTY, FOOD INSECURITY, AND PSYCHOSOCIAL STRESS AMONG AIDS CARE VOLUNTEERS IN URBAN ETHIOPIA. PMC. 2011;35(1):98-115.

21. JebenaMG L, BelachewT, and al. FoodlnsecurityandCommonMentalDisorders amongEthiopianYouth:StructuralEquation Modeling. PLoSONE. 2016;11(11).

22. Jing Sun MK, Falguni Patel, and et. al. Childhood Adversity and Adult Reports of Food Insecurity Among Households With Children. American Journal of Preventive Medicine. 2016 50(5):561-72.

23. Laraia BA. Food Insecurity and Chronic Disease. American Society for Nutrition Adv Nutr. 2013 4:20312.

24. ENZAGUCCIARDI J, MARGARETDEMELO, RD, and et. al. Exploration of the Relationship Between Household Food Insecurity and Diabetes in Canada Diabetes Care. 2009;32(12):2218-24.

25. : G. Persistent household food insecurity, HIV, and maternal stress in Peri-Urban Ghana. BMC Public Health. 2013 13(215).

26. Gedle DMG, Kumera G, and et. al. Food Insecurity and it's Associated Factors among People Living with HIV/AIDS Receiving Anti-Retroviral Therapy at Butajira Hospital, Southern Ethiopia. J Nutr Food Sci 2015 5(347).

27. Achenef Motbainor AWaAK. Household food insecurity is associated with both body mass index and middle upper-arm circumference of mothers in northwest Ethiopia: a comparative study. International Journal of Women's Health. 2017;9.

28. Holly C. Gooding CEWaTKR. Food Insecurity and Increased BMI in Young Adult Women. obesity journal 2012;20(9):1896-901.

29. Hartline-Grafton H. Understanding the Connections: Food Insecurity and Obesity. Food research and action center in 2015.

30. Barbara A. Laraia AMS-Raea. Household food insecurity is associated with self-reported pregravid weight status, gestational weight gain and pregnancy complications. J Am Diet Assoc. 2011;110(5): 692-701.

31. Samson Gebremedhin Ph.D. KBPD, Tilahun Bekele M.Phil, and et. al. Predictors of dietary diversity in children ages 6 to 23 mo in largely food-insecure area of South Wollo, Ethiopia. Elsevier IncNutrition 2017;33 163-8.

32. Belachew T LD, Gebremariam A, and et. al. Food Insecurity, Food-Based Coping Strategies and Suboptimal Dietary Practices of Adolescents in Jimma Zone Southwest Ethiopia. PLoS ONE. (2013); $8(3)$. 
33. Mariana Chilton MKSLB. The Intergenerational Circumstances of Household Food Insecurity and Adversity, Journal of Hunger \& Environmental Nutrition, 2016.

34. Markos Tesfaye PK, Mette Frahm Olsen, and et. al. Food insecurity, mental health and quality of life among people living with HIV commencing antiretroviral treatment in Ethiopia: a cross-sectional study. Health and Quality of Life Outcomes. 2016; 14(37).

35. Mohammed Feyisso TB, Amanuel Tesfay and et. al. Differentials of modern contraceptive methods used by food security status among married women of reproductive age in Wolaita Zone, South Ethiopia. Archives of Public Health. 2015;73(38).

36. health ECsaamo. Ethiopia Mini Demographic and Health Survey Central Statistical Agency of 2014.

37. Ethiopia CSA. Demographic and Health Survey 2016 Key Indicators Repor. DHS Program ICF Rockville, Maryland, USA 2016.

38. Zulfiqar Bhutta CCaHC. Nutrition country profile indicators: Definitions and sources. global nutrition report 2014.

39. Tadesse T. GA, Admassu M., Yigzaw Kebede Y., Awoke T., Tesfahun Melese T. Demographic and health survey at dabat district in northwest Ethiopia: report of the 2008 baseline survey. Ethiop $\mathrm{J}$ Health Biomed Sci, 2011; 4:1-23.

40. Bealu Betebo TE, Fissahaye Alemseged, and et.al. Household Food Insecurity and Its Association with Nutritional Status of Children 6-59 Months of Age in East Badawacho District, South Ethiopia. Hindawi Journal of Environmental and Public Health.Volume 2017.

41. Tesfay Birhane SS, Seifu Hagos2, and et. al. Urban food insecurity in the context of high food prices: a community-based cross-sectional study in Addis Ababa, Ethiopia. BMC Public Health 2014;14(680).

42. Tefera Belachew DL, Abebe Gebremariam, and et. al. Food Insecurity, Food-Based Coping Strategies and Suboptimal Dietary Practices of Adolescents in Jimma Zone Southwest Ethiopia. PLoS ONE 2013;8(3).

43. Helen Harris-Fry KA, Abdul Kuddus, and et. al. Socio-economic determinants of household food security and women's dietary diversity in rural Bangladesh: a cross-sectional study. Journal of Health, Population, and Nutrition. 2015;33(2).

44. Dessalegn Tamiru AA, Mulusew Gerbaba, and et. al. Household food insecurity and its association with school absenteeism among primary school adolescents in Jimma zone, Ethiopia. BMC Public Health. 2016;16(802).

45. Ayele Tiyou TB, Fisehaye Alemseged and et.al. Food insecurity and associated factors among HIVinfected individuals receiving highly active antiretroviral therapy in Jimma zone Southwest Ethiopia. Nutrition Journal. 2012;11(51).

46. Mulusew G. Jebena DL, and et. al. Food Insecurity and Common Mental Disorders among Ethiopian Youth: Structural Equation Modeling. PLoS ONE. 2016;11(11).

47. Mulusew G. Jebena DL, and et. al. The effect of food insecurity on the health status of adolescents in Ethiopia: a longitudinal study. BMC Public Health. 2017 17(465). 
48. Laura C Anderson Ayalew Tegegn FT, Sandro Galea, and Craig Hadley. Food insecurity, childhood illness and maternal emotional distress in Ethiopia. Public Health Nutrition. 2011;15(4):648-55.

49. Tefera Belachew1 $\mathrm{CH}$, David Lindstrom, and et. al. Food insecurity, school absenteeism and educational attainment of adolescents in Jimma Zone Southwest Ethiopia: a longitudinal study. Nutrition Journal. 2011;10(29).

\section{Figures}

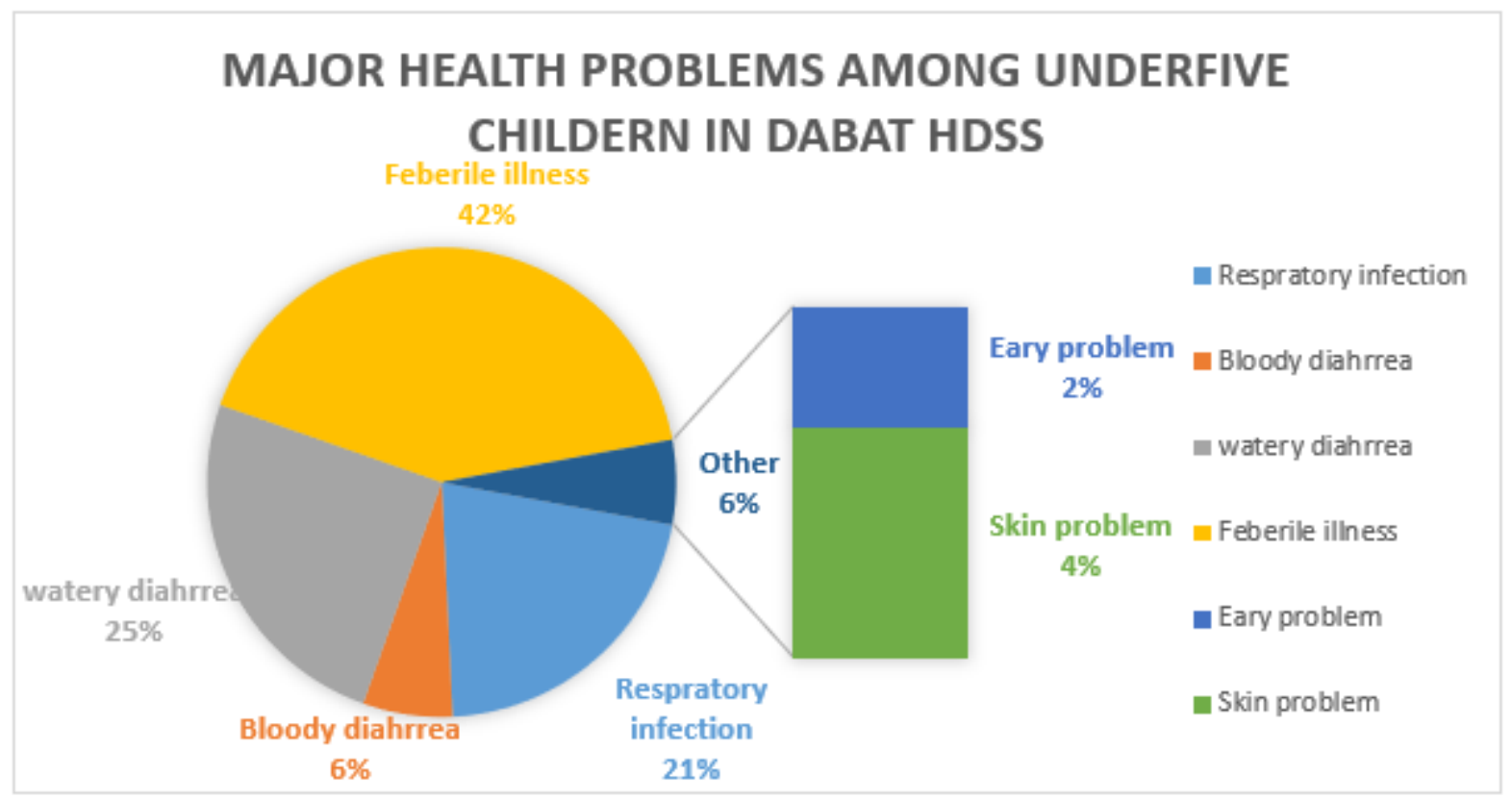

Figure 1

major child health problems among households included in the survey in Dabat district 


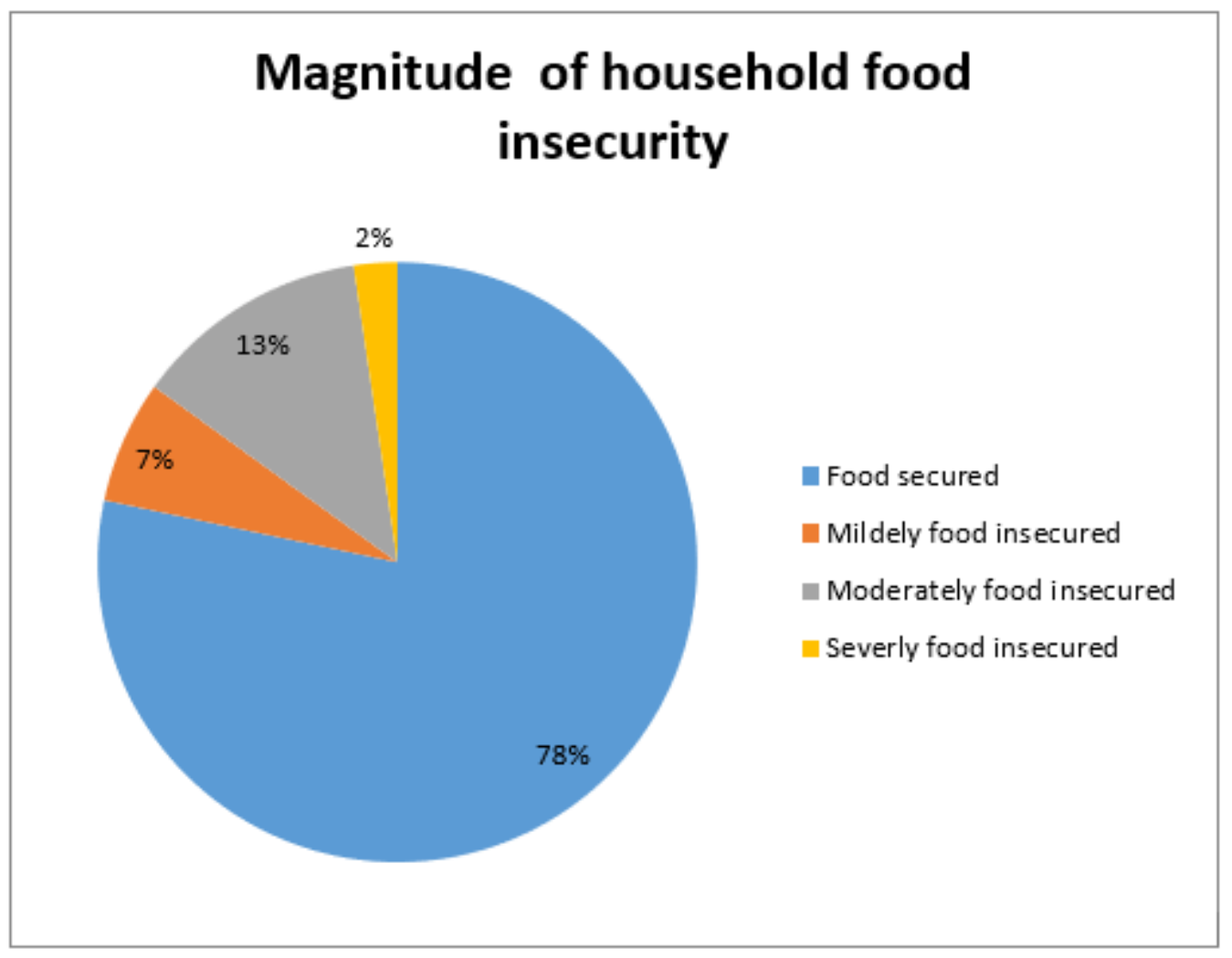

Figure 2

the magnitude of different levels of household food insecurity in Dabat district 


\section{Major food insecurity copying staregies}

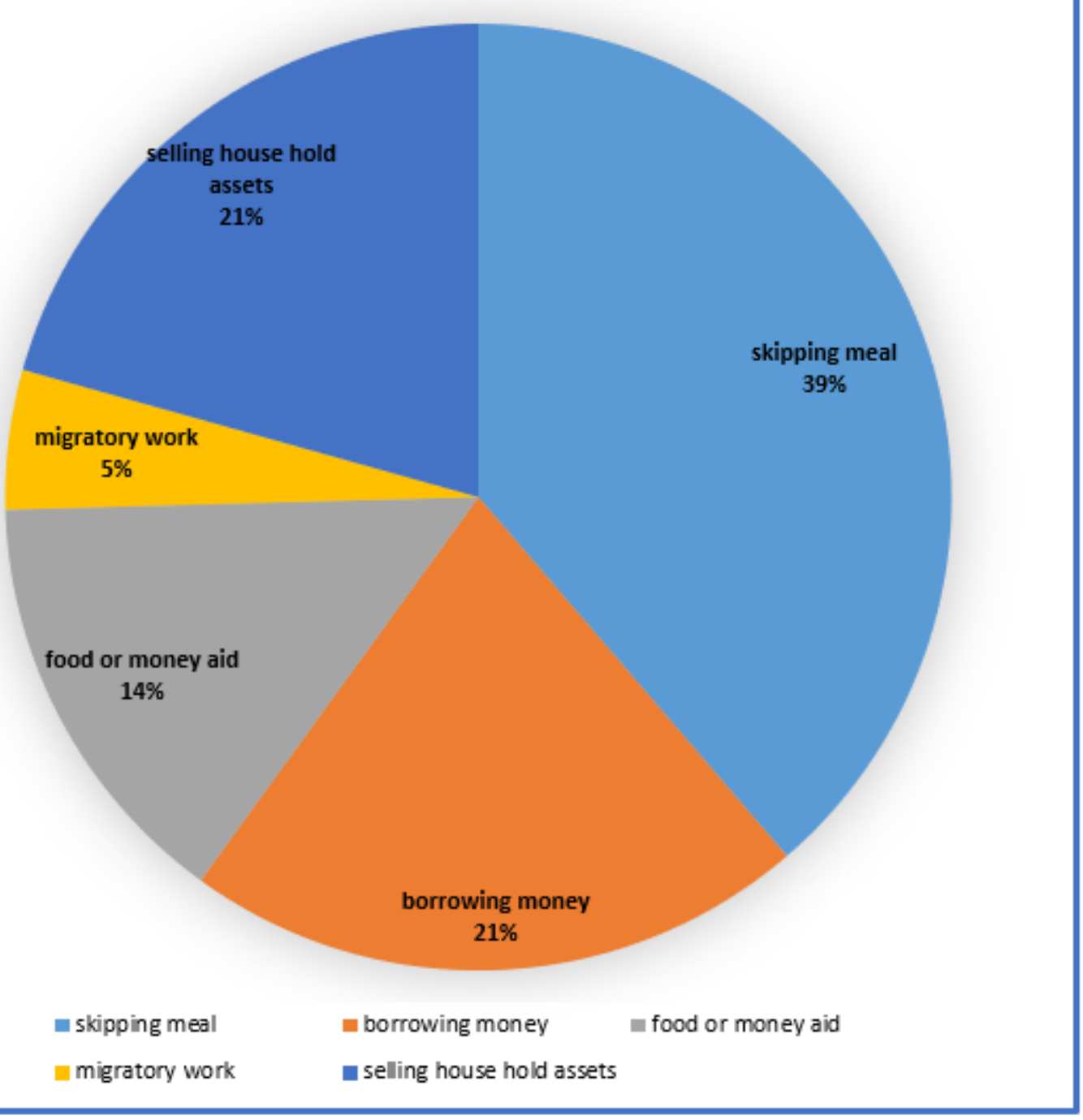

\section{Figure 3}

Identified household food insecurity coping strategies among households included in the survey.

\section{Supplementary Files}

This is a list of supplementary files associated with this preprint. Click to download.

- Tables.pdf 